\title{
Lidil
}

Revue de linguistique et de didactique des langues

49 | 2014

L'analyse des données didactiques

\section{Du concept de répertoire langagier et de sa transposition didactique}

On the Verbal Repertoire Concept and on Its Didactical Transposition

\section{Marion Dufour}

\section{(2) OpenEdition}

\section{Journals}

Édition électronique

URL : http://journals.openedition.org/lidil/3515

DOI : 10.4000/lidil.3515

ISSN : 1960-6052

Éditeur

UGA Éditions/Université Grenoble Alpes

\section{Édition imprimée}

Date de publication : 25 mai 2014

Pagination : 179-194

ISBN : 978-2-84310-272-1

ISSN : $1146-6480$

\section{Référence électronique}

Marion Dufour, « Du concept de répertoire langagier et de sa transposition didactique », Lidil [En ligne], 49 | 2014, mis en ligne le 25 novembre 2015, consulté le 30 avril 2019. URL : http://

journals.openedition.org/lidil/3515; DOI : 10.4000/lidil.3515

(c) Lidil 


\title{
Du concept de répertoire langagier et de sa transposition didactique
}

\author{
Marion Dufour*
}

\begin{abstract}
RÉSUMÉ
Dans cet article nous traitons de la transposition didactique externe du concept de répertoire langagier issu du champ de l'ethnographie de la communication. Nous attirons l'attention sur l'imprécision avec laquelle il a été décrit, sur le plan théorique, dans le CECR; élément qui, ajouté au bouleversement épistémologique qu'il induit dans le champ de la didactique du plurilinguisme, met au défi les enseignants de langues de se le représenter clairement et de se l'approprier. D'où résulte la nécessité d'approfondir la didactisation de ce concept.
\end{abstract}

\begin{abstract}
This article deals with the didactical transposition of the verbal repertoire concept, arising from the realm of ethnography of communication. It underlines theoretically gray areas surrounding the description of this concept in the CEFR, element which combined with the changes of perspective it has involved in the field of didactics for plurilingualism, challenges language teachers to have a clear image of this concept and to internalize it. Hence, the need to deepen the didactical transposition of this concept.
\end{abstract}

Au cours des dernières décennies, sous l'effet de la mobilité croissante des personnes, et du développement des réseaux de communication et des technologies de l'information, les contacts sociaux et linguistiques se sont accentués. Les individus sont aujourd'hui confrontés, au quotidien, à une réalité sociolinguistique complexe qui dépasse de loin les images stéréotypées construites avec l'histoire des peuples (conflits politiques et économiques, proximité géographique, échanges commerciaux, sociaux, scolaires, etc.), sur lesquelles ils ont fondé leurs représentations des langues et de leurs locuteurs.

* Enseignante de français, King's College London. 
Pour, d'une part, encourager les rencontres entre individus de langues premières différentes et accompagner ces expériences intersubjectives susceptibles d'ébranler voire de modifier leurs représentations, et, d'autre part, sauvegarder et valoriser le patrimoine que représente la diversité linguistique et culturelle en Europe, le Conseil de l'Europe a fait le choix de fonder ses politiques linguistique et d'enseignement des langues sur le principe du plurilinguisme. En didactique des languescultures, le choix du plurilinguisme signifie : faire en sorte que tous les individus développent une «compétence potentielle et/ou effective à utiliser plusieurs langues, à des degrés de compétence divers et pour des finalités différentes» (Beacco \& Byram, 2003, p. 8). Cette formation va de pair avec l'éducation au plurilinguisme qui consiste à inciter les individus à aller à la rencontre les uns des autres, à les sensibiliser à la diversité des langues et des cultures étrangères et à les former à la citoyenneté démocratique (ibid., 2003, p. 16). Dans la perspective de la mise en œuvre de cette politique, une équipe de chercheurs européens a mené une réflexion de 1991 à 2001 qui a abouti à l'élaboration du Cadre européen commun de référence pour les langues (désormais CECR), commun aux 47 membres du Conseil de l'Europe et largement diffusé à travers le monde.

Étant donné les enjeux de cohésion sociale sous-tendus par cette politique, l'élaboration de ce cadre particulier a nécessité la redéfinition des concepts clés de la didactique des langues dans une perspective sociale. Ainsi, considérant d'une part que tout individu est un sujet énonciateur qui endosse une posture (voir Portine, 2010, p. 126) et d'autre part qu'apprendre n'est pas un processus passif, mais un processus dynamique d'appropriation et de transformation des connaissances (Vygotski, 1997 [1934]), usagers et apprenants d'une langue ont été redéfinis comme «des acteurs sociaux ayant à accomplir des tâches (qui ne sont pas seulement langagières) dans des circonstances et un environnement donnés, à l'intérieur d'un domaine d'action particulier» (CECR, 2000, p. 11); l'acte de parole, concept clé de l'approche communicative, a été repensé en relation avec le contexte social dans lequel il apparait; la langue a été redéfinie comme constitutive d'un répertoire langagier dans lequel toutes les langues sont en corrélation et interagissent (CECR, 2000, p. 11); enfin, la manière de concevoir le but de l'éducation aux langues a été également modifiée : il s'agit de «développer un répertoire langagier dans lequel toutes les capacités linguistiques trouvent leur place» (CECR, 2000, p. 11). Ce nouveau but de l'éducation aux langues va de pair avec le nouvel objectif social 
de référence que l'on vise qui n'est plus, comme dans les années 19801990, de favoriser la mobilité à l'intérieur de l'Europe dans le cadre de voyages et d'échanges ponctuels, soit touristiques, soit professionnels, mais de concevoir que tout apprenant pourra être amené, à différents moments de sa vie, à effectuer plusieurs séjours longs à l'étranger, pour y faire une partie de ses études et/ou une partie de sa carrière en langue étrangère.

Étant donné le rôle beaucoup plus actif donné à l'apprenant et le nouveau but de l'éducation aux langues, la posture et la mission de l'enseignant de langues se trouvent, elles aussi, inévitablement modifiées et consistent aujourd'hui à accompagner l'apprenant dans le développement d'une compétence plurilingue et pluriculturelle (Coste, Moore \& Zarate, 2009; Castellotti \& Moore, 2011), caractérisée comme «la capacité à mobiliser et à faire évoluer les ressources langagières d'un répertoire (hétérogène) en vue de leur mise en œuvre dans des familles de situations à des fins de résolutions de tâches et d'interaction sociale» (Coste, 2010, p. 150). Le caractère global et non segmenté du répertoire langagier (désormais RL) et la dimension dynamique et individualisée d'une compétence plurilingue unique qui englobe l'ensemble du répertoire à disposition induisent un bouleversement épistémologique qui soulève la question des représentations que les enseignants de langues ont des langues et de leur enseignement/apprentissage, et celle de leur formation à ces nouveaux concepts.

C'est dans cette perspective que nous avons investigué le concept de RL, désormais matière sur laquelle enseignants et apprenants travaillent collectivement. Ce travail de recherche nous a conduite à proposer cet essai réflexif dans lequel nous montrons, dans une première partie, que le concept de RL semble insuffisamment établi en didactique des langues, ce qui nous amène, dans une seconde partie, à nous intéresser au processus de didactisation (Chevallard, 1991) qu'il a suivi, c'est-à-dire à la chaine de transposition didactique qui va de la connaissance scientifique que les spécialistes ont de cet objet jusqu'aux savoirs appropriés par les enseignants de langues en vue de leur pratique d'enseignement. Cependant, cette seconde partie fera uniquement l'objet de ce que Chevallard a nommé la «transposition didactique externe», c'est-à-dire la chaine scientifique qui va de l'état de savoirs savants à l'état de savoirs à enseigner. 


\section{Le répertoire langagier, un concept à expliciter en didactique des langues}

Les recherches que nous avons entreprises sur le RL nous ont permis d'observer que l'appellation que nous avions retenue de «répertoire langagier», qui reprend celle que l'on trouve dans le CECR, ne faisait pas l'unanimité chez les chercheurs en didactique des langues et dans les disciplines connexes. En effet, comme l'a déjà mentionné Rosen (2005, p. 123), «plusieurs dénominations peuvent être rencontrées pour désigner ce répertoire : "répertoire communicatif" pour Dabène (1994, p. 153), "répertoire pluriel et dynamique" pour Lüdi (2000, p. 181), "répertoire plurilingue" pour Murphy-Lejeune \& Zarate (2003, p. 37) ou encore "répertoire linguistique" pour Coste (2002, p. 117)». Le fait que ces chercheurs de renom ne se soient pas mis d'accord concernant le nom de l'objet commun sur lequel ils travaillent, soulève une interrogation et semble traduire un certain flottement théorique autour de cette notion. Dépassant la question de sa dénomination, nous nous sommes penchée sur la définition du RL proposée dans le CECR, outil de référence des professionnels de l'enseignement/apprentissage des langues, afin de mieux comprendre ce dont il retourne. Dès le premier chapitre, les auteurs du Cadre font allusion à la construction, à la composition, à la structure et à l'utilisation du répertoire langagier, à travers les lignes suivantes :

L'expérience langagière d'un individu dans son contexte culturel s'étend de la langue familiale à celle du groupe social puis à celles d'autres groupes (que ce soit par apprentissage scolaire ou sur le tas), il/elle ne classe pas ces langues et ces cultures dans des compartiments séparés mais construit plutôt une compétence communicative à laquelle contribuent toute connaissance et toute expérience des langues et dans laquelle les langues sont en corrélation et interagissent. (CECR, 2000, p. 11)

Ce paragraphe permet aux lecteurs du CECR de comprendre tout d'abord que le RL d'un individu se développe au fur et à mesure que celui-ci traverse différents domaines de l'activité humaine et que son expérience langagière se diversifie. Il est donc évolutif et variable suivant les moments de sa vie langagière, caractéristiques qui ne remettent pas en question sa compétence de communication, comme l'a clairement expliqué Grosjean (1993) :

L'être communiquant peut varier d'un monolinguisme fonctionnel à un autre, en passant par des étapes de bilinguisme, tout en préservant la même compétence communicative de base. Les changements de mi- 
lieux, de besoins, de situations feront que cette personne aura à restructurer sa compétence langagière; ils n'auront par contre aucun effet sur sa compétence communicative - celle-ci restera la même tout au long de cette restructuration. (p. 17)

Tout cela suggère que le RL est caractérisé par une grande plasticité, qu'il faut relier à l'activité neuronale, au fonctionnement de la mémoire et aux modifications des réseaux neuronaux que sous-tend tout nouvel apprentissage.

Concernant la structure du RL, le paragraphe du CECR cité plus avant énonce que «les langues ne sont pas classées dans des compartiments séparés $[\ldots]$ mais sont en corrélation et interagissent ensemble» (CECR, 2000, p. 11). Cette description émane des recherches issues des domaines de la sociolinguistique et de la linguistique de l'acquisition des langues secondes - nous y reviendrons - qui, dans les années 1980, ont permis de caractériser les usages que les individus font de leurs ressources langagières (procédés d'alternance codique), et les stratégies d'accommodation qu'ils adoptent dans le processus qui leur permet de s'intercomprendre. Plus récemment, les recherches en neuropsycholinguistique (Kim et coll., 1997; Perani et coll., 1998; Fabbro \& Paradis, 1995; Fabbro, 2001) ont infirmé l'hypothèse selon laquelle deux langues bien maitrisées seraient sous-tendues par des aires cérébrales distinctes, les zones activées par les différentes langues se recouvrant quasiment parfaitement chez les bilingues de haut niveau et partiellement chez les bilingues «non parfaits». Dans cette perspective, le RL apparait donc comme une métaphore pratique utilisée pour traiter du système de communication ancré dans le corps et dans le cerveau des individus, grâce aux capacités générales de perception, d'action, d'apprentissage, de raisonnement, de mémoire et d'attention qu'ils développent. Il permet de rassembler sous une même étiquette des informations et des processus qui mettent en jeu des régions cérébrales plus ou moins définies (lobes frontal, temporal, occipital, pariétal, gyrus angulaire, etc.) qui fonctionnent dans le cadre de réseaux neuronaux qui les mettent en relation les unes avec les autres.

Concernant la composition du répertoire, le paragraphe du CECR indique qu'il est composé de langues : «langue(s) familiale(s) puis langues d'autres groupes sociaux traversés », et de cultures, notion non explicitée. Le mot «langues» dans cette définition semble donc devoir être compris au sens de «sociolectes» qui peuvent être issus de systèmes linguistiques divers. Dans une autre définition, Coste (2002) préfère au mot «langue» celui de «variétés linguistiques» : 
Le concept de répertoire linguistique par lequel les sociolinguistes caractérisent l'ensemble de variétés linguistiques - plus ou moins bien maîtrisées ou développées - que possède un acteur social donné et dont il peut jouer, selon les situations, en faisant appel à telle ou telle de ces variétés, est solidement établi et a servi aussi la réflexion didactique [...]. (p. 117)

On notera que dans cette définition où le RL est constitué uniquement de «variétés linguistiques», ce terme doit être pris dans son acception sociolinguistique, désignant alors différentes «façons de parler» (Gadet, 2007 [2003], p. 13). Cependant, il apparait que les termes de «langues » et de «variétés linguistiques » sont problématiques pour désigner les ressources contenues dans l'espace interactif du répertoire. En effet, le mot «langue», largement polysémique, ouvre la porte à de multiples interprétations de cette définition, selon le sens que le lecteur donne à ce mot (langue-standard, langue-nationale, langue-système de dialectes, etc.) et peut donc conduire à des représentations fausses du RL; ce terme n'est, en outre, pas assez explicite pour permettre au lecteur de comprendre que les représentations conceptuelles (ou sémantiques), lexicales, et phonologiques qui accompagnent la production de la parole, le traitement du signal et les processus interprétatifs, font partie de cet objet étroitement lié au psychisme des individus. Quant au mot «variété», il a l'inconvénient de prêter à confusion en laissant suggérer que le RL est constitué d'un ensemble de parlers homogènes bien délimités, découpage qui, comme le souligne Gadet (2007), «ne résiste pas à l'observation des productions effectives qui peuvent être souples, labiles et plus souvent hétérogènes qu'homogènes » (p. 22). Notons que cette représentation structurale simpliste d'un ensemble cloisonné de «langues » est confortée par le terme de «répertoire» lui-même, fréquemment utilisé dans le sens de «recueil où les matières sont rangées dans un ordre qui les rend faciles à trouver (répertoire alphabétique, répertoire de métier)»(Petit Larousse illustré, 2005, p. 921). Plutôt qu'un répertoire, cet ensemble semble davantage évoquer ce que l'on appelle en langage informatique un cloud, c'est-à-dire un espace où sont emmagasinées des ressources, qui interagiraient les unes avec les autres au sein d'un réseau plus large et pourraient se combiner de multiples manières. Cela nous amène à suggérer l'usage de «cloud langagier» en place de celui de RL.

L'imprécision terminologique se poursuit avec l'emploi du terme non explicité de «culture», sous lequel il faut entrevoir non seulement la culture langagière de l'individu, c'est-à-dire les représentations sociales 
langagières qu'il s'est construites des activités langagières constitutives de son environnement social, et qui sont relatives aux connaissances qu'il a des règles socioculturelles et sociolinguistiques qui gouvernent les usages : «qui peut prendre part à la conversation, quels sont les rapports des rôles entre eux, quel type de contenu est autorisé, dans quel ordre l'information doit-elle être introduite et quelles sont les convenances relatives à la pratique langagière» (Gumperz, 2005 [1982], p. 57), mais encore les processus au moyen desquels il s'est approprié les savoirs langagiers qu'il a expérimentés, qui ont à voir avec les domaines de la culture éducative et de la culture didactique (Beacco, Chiss, Cicurel \& Véronique, 2005); ce qui revient à dire que le RL contient en plus d'un répertoire linguistique, d'une part un répertoire de représentations sociales langagières de référence et d'usage (Py, 2004, p. 10) qui interviennent dans l'apprentissage sous la forme de croyances, de connaissances et de modèles, et d'autre part un répertoire d'aptitudes psycho-cognitives qui prend appui sur le développement de stratégies cognitives et métacognitives, de connaissances déclaratives, procédurales et conditionnelles, et de modèles métalinguistiques (voire le développement d'une culture métalinguistique). Sous l'appellation de RL, il faudrait donc entrevoir un cloud langagier dynamique constitué d'un ensemble de «répertoires» interactifs sur lesquels l'individu s'appuie pour développer sa compétence plurilingue et pluriculturelle.

In fine, on s'aperçoit, après analyse, que le caractère beaucoup trop évasif de la définition du CECR sur le plan théorique, et le parti pris d'ancrer le concept de RL uniquement dans une dimension sociolinguistique, au détriment de sa dimension psycho-cognitive, en offrent une représentation lacunaire. On peut, en outre, s'interroger sur ce choix à une époque où la question des relations entre langage et cognition mobilise l'intérêt de nombreux chercheurs sur les plans national et international, et où le domaine de l'ingénierie du langage et des apprentissages est en pleine expansion. Ainsi, cette définition permet difficilement à l'enseignant de langues, soucieux d'être au fait du concept de RL, de comprendre d'une part ce qui le caractérise et d'autre part en quoi son introduction en didactique des langues permet une nouvelle donne : «Il ne s'agit plus simplement d'acquérir la "maîtrise" d'une, deux, voire même trois langues, chacune de son côté, avec le "locuteur natif idéal" comme ultime modèle» (CECR, 2000, p. 11). Elle soulève en conséquence des interrogations quant au processus de transposition didactique externe suivi par ce concept, auquel nous allons nous intéresser dans une seconde partie, en examinant dans un premier temps 
l'émergence du concept de RL en ethnographie de la communication et dans un deuxième temps son introduction en didactique des langues.

\section{De la transposition du concept de RL de l'ethnographie de la communication à la didactique des langues}

\section{Le RL en ethnographie de la communication : un ensemble de styles}

Le concept de RL est issu de l'ethnographie de la communication, champ ouvert au début des années 1960 par Hymes et Gumperz, et qui se donne pour «objectif ultime l'analyse de la compétence communicative, c'està-dire un savoir cognitif abstrait» (Gumperz, 2005, p. 57). On doit plus particulièrement la formulation du concept de RL à Gumperz qui l'a explicité dans un article de 1964 intitulé «Linguistic and Social Interaction in Two Communities ». Dans cet article, Gumperz (1971) désigne par répertoire langagier «la totalité des formes linguistiques régulièrement employées au cours d'interactions socialement significatives ${ }^{1}{ }^{\circledR}$ (p. 152) dans laquelle chaque individu fait des choix selon les intentions qu'il souhaite communiquer. En parlant «d'interaction socialement significative», Gumperz véhicule une représentation sociolinguistique du langage qui consiste en une organisation sociale. Il faut, en effet, comprendre par «interaction» l'ensemble de l'interaction qui se produit au cours d' ' activités ou parties des activités directement régies par les règles ou par les normes d'usage de la parole» (Hymes, 1980 [1972], p. 135). Cette représentation est innovante à une époque où les études linguistiques sont davantage orientées vers le système que vers l'utilisation de ce système. Gumperz explique dans The Ethnography of Communication (1972, p. 20-21) qu'il a introduit le concept sociolinguistique du RL pour rendre compte du fait que l'analyse grammaticale des linguistes de l'époque correspond rarement au comportement langagier des populations dans la réalité. Ce concept va donc de pair avec un nouveau point de vue porté sur les phénomènes langagiers d'une communauté sociolinguistique et sur leur locuteur : les langues et dialectes sont considérés comme des produits socio-historiques, et non

1. «Procedures such as these enable us to isolate the verbal repertoire, the totality of linguistic forms regularly employed in the course of socially significant interaction.» 
plus comme des organismes ayant leur vie propre, tandis qu'une place est redonnée au sujet énonciateur et au contexte social dans lequel il interagit.

Pour comprendre comment se font les choix langagiers au sein du RL au cours des interactions, Gumperz (1971) est parti de l'idée que le RL devait avoir une structure interne : «Dans la mesure où la communication orale peut être décrite par un ensemble fini de règles qui sous-tendent la formation de toutes phrases, les répertoires langagiers doivent avoir une structure ${ }^{2}$.» (p. 152) L'analyse d'interactions recueillies au cours de deux grandes enquêtes de terrain dans les communautés sociolinguistiques de Hemnes en Norvège et de Khalapur en Inde va lui permettre de confirmer cette hypothèse. En effet, en examinant plus particulièrement les restrictions de cooccurrence qui s'appliquent aux évènements langagiers («speech events» chez Hymes) auxquels ont pris part les participants, il constate que les choix langagiers affectent toutes les strates linguistiques, conditionnant ainsi simultanément les réalisations morphologiques et phonologiques des messages. Cette étude le conduit à conclure que les règles de cooccurrence définissent des «variétés langagières» («speech varieties» dans le texte), et que les individus font leurs choix langagiers compte tenu des «variétés langagières » qui structurent leur répertoire (voir Gumperz, 1971, p. 156), autrement dit de «dialectes» («variétés» associées à des groupes sociaux) et de «styles» («variétés» associées à des situations de communication) (voir Gumperz, 2005, p. 57).

Dans son ouvrage intitulé Vers la compétence de communication, Hymes (2009, p. 59) préfère au terme de «variétés linguistiques» celui de «styles de parole» («speech styles») pour traiter des «moyens de la parole» («means of speaking»). Par «styles de parole» il distingue deux ensembles principaux : «les principaux styles de parole associés à des groupes sociaux qui peuvent être dénommés variétés; et les principaux styles associés à des types récurrents de situations [qui] peuvent être dénommés registres» (ibid., p. 60), ce qui rejoint la description de Gumperz. Parler de «dialectes» et de «styles», comme chez Gumperz, ou bien de «variétés » et de «registres » comme chez Hymes, plutôt que de «langues», permet de bien saisir que le concept de RL offre un

2. «Since spoken communication of all kinds is describable by a finite set of rules which underlie the formation of all possible sentences, verbal repertoires must have structure.» 
cadre conceptuel permettant de traiter d'individus de tous types, qu'ils soient «monolingues» ou «plurilingues», le plurilinguisme comme le monolinguisme renvoyant chacun à un ensemble de styles de parole.

Selon Gumperz et Hymes, les styles pertinents d'une communauté peuvent être mis en évidence par l'analyse des contextes. Hymes (2009) dit aussi que :

[...] le critère d'identification d'un style de parole significatif est qu'il peut être reconnu et utilisé en dehors du contexte qui le définit, c'està-dire par des individus ou dans des lieux autres que ceux auxquels est associée sa signification type; c'est ainsi qu'il peut se trouver en contraste avec un ou plusieurs autres styles quant aux individus et aux lieux. (p. 60)

Il pose ainsi d'une part que les styles de parole sont marqués par la spécificité de sphères d'échange («on peut déterminer les styles associés à des castes, des classes, groupes ethniques, régions » [Hymes, 2009]) et par les genres de discours (discours oratoire, sermon) auxquels ils sont liés (voir Hymes, 1980 [1967], p. 146), et d'autre part que les membres d'une communauté sociolinguistique ont en partage un savoir linguistique (normes de grammaire) et un savoir sociolinguistique (normes d'emploi) qui leur permettent de faire un usage créatif de la parole (Hymes, 2009, p. 47). Il rejoint en ce sens la réflexion de Bakhtine (1984), qui passe pour un des premiers à avoir proposé une représentation du langage consistant en une organisation sociale :

$[\ldots]$ chaque sphère connait ses genres, appropriés à sa spécificité, auxquels correspondent des styles déterminés [...] quand on fait passer le style d'un genre à un autre on ne se borne pas à modifier la résonance de ce style à la faveur de son insertion dans un genre qui ne lui est pas propre, on fait éclater et on renouvelle le genre donné. (p. 269-271)

Mais Hymes (2009) ne limite pas son analyse à l'usage et à la fonction des styles, il pose également, comme d'autres avant lui, la question de l'existence de «styles cognitifs» liés aux capacités internes des individus, tout en restant très prudent sur ce sujet comme en témoignent les lignes suivantes :

Ce que j'affirme, c'est que l'importance des styles cognitifs en matière de langue est problématique, qu'elle a besoin d'être établie; et ceci ne revient aucunement à nier cette importance. Il en va de même pour tous les styles de parole et pour les moyens de parole en général. (p. 71) 
En parlant de «style cognitif», il rejoint d'une certaine manière encore une fois Bakhtine (1984) qui a considéré les genres de discours et les styles comme des outils sémiotiques, des représentations sociocognitives (Moirand, 2003) partagées que les individus intériorisent relativement aux sphères d'activités qu'ils traversent au cours de leur vie, et au moyen desquelles ils construisent, anticipent et interprètent les activités langagières à l'intérieur d'une situation de communication :

[...] les formes de la langue et les formes de types d'énoncés, c'est-àdire les genres du discours, s'introduisent dans notre expérience et dans notre conscience conjointement et sans que leur corrélation étroite soit rompue. [...] Nous apprenons à mouler notre parole dans les formes du genre et, entendant la parole d'autrui, nous savons d'emblée, aux tout premiers mots, en pressentir le genre, en deviner le volume, la structure compositionnelle donnée, en prévoir la fin, autrement dit, dès le début, nous sommes sensibles au tout discursif. (Bakhtine, 1984, p. 285)

Ainsi, l'expression d'un style de parole pour Gumperz et Hymes est relative à un ensemble de connaissances linguistiques (lexique, phraséologie, grammaire), sociolinguistiques (normes grammaticales et normes d'usage), et de représentations sociolangagières et sociocognitives dont dispose un acteur social.

En conclusion, dans la définition du RL que l'on trouve dans le CECR, les termes utilisés sont ceux de «langues et de cultures», et non les termes retenus en ethnographie de la communication (styles de paroles) pour traiter de la structure du RL. Ce constat semble indiquer que les auteurs du Cadre ne se sont pas référés directement à ce champ de recherche pour construire leur définition, bien qu'ils aient fait le choix d'une approche sociolinguistique. Cela nous conduit à postuler que le concept de RL - contrairement au concept de compétence de communication, également issu de ce champ disciplinaire - a suivi un processus de didactisation qui ne s'est pas fait de manière descendante, c'est-à-dire dans une visée applicationniste du concept de RL établi en ethnographie de la communication vers la didactique des langues.

\section{L'émergence du RL en didactique des langues}

Les concepts de RL et de compétence de communication sont intimement liés : le premier pose la question des ressources langagières et cognitives dont dispose un acteur social, et de leur organisation, et le second pose celle de ses connaissances et de ses capacités en vue de la 
mobilisation de ces ressources pour interagir en société de façon appropriée. En didactique des langues, l'introduction de ces concepts va de pair avec le développement de l'approche communicative, approche élaborée dans les années 1970-1980, sur l'analyse d'observation de classes, en milieu scolaire, regroupant des enfants de migrants et sur l'analyse de leurs interactions dans une approche acquisitionnelle de la langue d'une part et sociolinguistique d'autre part, traversée par un courant interactionniste fort. En effet, l'appropriation de la langue y est pensée en termes acquisitionnels : on part du principe que, comme en langue maternelle, c'est en communiquant que l'on apprend la langue (l'interaction est constitutive de l'acquisition), et en termes de socialisation en s'appuyant sur l'idée que c'est par l'immersion dans des contextes sociaux de communication que l'on apprend à interagir. Dans l'approche communicative, la langue est considérée de manière fonctionnelle comme un outil de communication permettant de s'exprimer et de réagir au cours de situations de communication ponctuelles au sein de domaines divers (professionnels, touristiques, familiaux, commerciaux, etc.). De ce fait, la notion de compétence de communication connait vite un grand succès auprès des didacticiens des langues, promouvant l'approche communicative dans le contexte de la construction de l'Europe et de son développement économique (ouverture de ses marchés) où les échanges ponctuels, qu'ils soient touristiques ou professionnels, s'intensifient. Les différentes taxonomies (voir Canale \& Swain, 1980; voir aussi Bachman, 1990 et Bachman \& Palmer, 1996) dont cette notion fait l'objet participent de sa didactisation.

Outre le fait que l'introduction de la notion de compétence de communication en didactique des langues entraine la prise en compte de stratégies cognitives, de savoirs fonctionnels, sociolinguistiques et discursifs, ce qui n'était pas le cas jusque-là, elle s'accompagne également de l'idée véhiculée dans le domaine de la psychologie cognitive par Piaget et Vygotski selon laquelle la compétence de communication en langue seconde ou étrangère se développe sur des savoirs et sur une compétence déjà existants. Dans les années 1980, des enquêtes psycholinguistiques qui s'intéressent, d'une part, aux modes d'acquisition des langues à travers notamment les mécanismes de l'interaction exolingue et, d'autre part, aux processus de construction de l'interlangue (Corder, 1980 ; Noyau \& Porquier, 1984; Py \& Alber, 1984), corroborent cette idée. Ces travaux amènent, en effet, les chercheurs à considérer que les productions de l'apprenant sont conçues comme des manifestations d'un système transitoire qu'il construit sur la base de son expérience 
et de ses connaissances en langue première. Parallèlement, dans les domaines de l'acquisition des langues et de la sociolinguistique, les recherches sur le parler bilingue concernant, notamment, la manière dont le bilingue utilise les ressources langagières qui constituent son répertoire au quotidien, mettent en évidence le lien entre choix de langue et contexte situationnel et l'asymétrie des compétences dans les différentes langues du locuteur bilingue (Lüdi, 1987; De Heredia, 1987). Toutes ces recherches permettent ainsi d'aboutir à une nouvelle conception du bilinguisme : auparavant défini de manière restrictive comme la capacité d'un individu à maitriser parfaitement deux ou plusieurs langues, il est ensuite considéré sous l'angle de la compétence communicative d'un individu face à ses besoins de tous les jours : «Le bilingue n'est pas deux monolingues mais un tout qui a sa propre compétence linguistique et qui doit être analysé en tant que tel.» (Grosjean, 1993, p. 16)

Dans cette perspective, l'acquisition d'une langue seconde ou étrangère peut être considérée comme un cas particulier de bilinguisme (Py, 1991, p. 160). De ces considérations nait le champ de la didactique du plurilinguisme, qui considère les potentialités d'un apprenant en termes de répertoire langagier constitué de différentes formes de pluralités : "pluralité des ressources linguistiques, des représentations, des contextes, des stratégies et compétences, qui se distribuent et évoluent dans l'espace et dans le temps» (Castellotti \& Moore, 2005, p. 109). Le concept de RL, tel que défini par les ethnographes de la communication (ensemble de styles de paroles), s'étaye ainsi d'une dimension représentationnelle et d'une dimension psycho-cognitive, qui permettent à Castellotti et Moore (2005) de le définir comme constitué non seulement «par les usages que les locuteurs peuvent déployer, mais aussi par l'ensemble des représentations et aptitudes susceptibles de générer ces usages ou de les accompagner» (p. 111).

En conclusion, la transposition didactique du concept de RL n'a pas été effectuée dans une visée applicationniste du concept de RL établi en ethnographie de la communication, mais par le biais de recherches empiriques menées, dans les domaines de l'acquisition, de la psycholinguistique et de la sociolinguistique, sur les interactions exolingues et bilingues, dans le souci d'étudier le rôle des langues dans le mécanisme de l'interaction, leurs fonctions, les usages qu'en font les interactants, c'est-à-dire essentiellement sous l'angle de leur compétence plurilingue. Ces recherches ont permis de préciser la définition que les ethnographes de la communication avaient donnée du RL en y incluant les aptitudes 
psycho-cognitives et les représentations sociolangagières sur lesquelles les individus s'appuient pour développer leur compétence plurilingue et pluriculturelle, dimensions qui n'apparaissent pas clairement dans la définition retenue par le CECR. En conséquence, l'imprécision de cette définition, ajoutée au bouleversement épistémologique que le RL induit en didactique des langues, met au défi les enseignants de langues de se le représenter clairement et de se l'approprier, et constitue donc un écueil dans leur démarche de transposition interne (état de savoirs à enseigner en savoirs enseignés). De ce fait, un approfondissement de la didactisation du RL apparait nécessaire pour la formation des enseignants à une didactique du plurilinguisme, dans une approche intégrée du langage qui allie linguistique, sociolinguistique, psycholinguistique et neuropsycholinguistique.

\section{RÉFÉRENCES BIBLIOGRAPHIQUES}

BaChman, Lyle F. (1990). Fundamental Considerations in Language Testing. Oxford : Oxford University Press.

Bachman, Lyle F. \& Palmer, Adrian S. (1996). Language Testing in Practice: Designing and Developing Useful Language Tests. Oxford : Oxford University Press.

B AKhtine, Mikhaïl. (1984). Esthétique de la création verbale (éd. 1979). Paris : Gallimard.

BeAcCo, Jean-Claude \& B yram, Michael. (2003). Guide pour l'élaboration de politiques linguistiques éducatives en Europe. De la diversité linguistique à l'éducation plurilingue. Strasbourg : Conseil de l'Europe.

Beacco, Jean-Claude, Chiss, Jean-Louis, Cicurel, Francine \& Véronique, Daniel. (2005). Les cultures éducatives et linguistiques dans l'enseignement des langues. Paris : PUF.

Canale, Michael \& Swain, Merrill. (1980). Theoretical Bases of Communicative Approaches to Second Language Teaching Testing. Applied Linguistics, 1, 1-47.

Castellotti, Véronique \& Moore, Danièle. (2005). Répertoires pluriels, culture métalinguistique et usage d'appropriation. Dans J.-C. Beacco, J.-L. Chiss, F. Cicurel \& D. Veronique (dir.), Les cultures éducatives et linguistiques dans l'enseignement des langues (p. 107-132). Paris : PUF.

Castellotti, Véronique \& Moore, Danièle. (2010). Valoriser, mobiliser et développer les répertoires plurilingues et pluriculturels pour une meilleure intégration scolaire. Dans D. Little (dir.), Intégration linguistique et éducative des enfants et des adolescents issus de l'immigration. 
Document d'orientation (Études et ressources, no 4, p. 5-29). Strasbourg : Conseil de l'Europe.

Castellotti, Véronique \& Moore, Danièle. (2011). La compétence plurilingue et pluriculturelle. Genèses et évolutions. Dans P. Blanchet \& P. Chardenet (dir.), Guide pour la recherche en didactique des langues et cultures. Approches contextualisées (p. 241-252). Paris : Éditions des Archives contemporaines.

Chevallard, Yves. (1991). La transposition didactique : du savoir savant au savoir enseigné (éd. 1985). Grenoble : La Pensée sauvage.

Conseil de L'Europe. (2001). Un Cadre européen commun de référence pour les langues : apprendre, enseigner, évaluer. Paris : Didier.

Corder, S. Pit. (1980). Que signifient les erreurs des apprenants? Langages, $57,9-15$.

Coste, Daniel. (2002). Compétence à communiquer et compétence plurilingue. Notions en Questions, 6, 115-123.

Coste, Daniel. (2010). Diversité des plurilinguismes et formes de l'éducation plurilingue et interculturelle. Les Cahiers de l'Acedle, 7(1), 141165.

Coste, Daniel, Moore, Danièle \& Zarate, Geneviève. (2009). Compétence plurilingue et pluriculturelle. Vers un Cadre européen commun de référence pour l'enseignement et l'apprentissage des langues vivantes. Études préparatoires (éd. 1997). Strasbourg : Conseil de l'Europe.

DABÈNE, Louise. (1994). Repères sociolinguistiques pour l'enseignement des langues. Paris: Hachette.

DABÈne, Louise \& BILliez, Jacqueline. (1984). Recherches sur la situation sociolinguistique des jeunes issus de l'immigration. Grenoble : Centre de didactique des langues, Université de Grenoble.

De Heredia, Christine. (1987). Du bilinguisme au parler bilingue. Dans G. Vermes \& J. Boutet (dir.), France, pays multilingue (t. 2, p. 91-127). Paris : L'Harmattan.

FABBro, Franco. (2001). The Bilingual Brain: Cerebral Representation of Languages. Brain and Language, 79, 211-222.

FABBro, Franco \& PARAdIs, Michel. (1995). Differential Impairments in Four Multilingual Patients with Subcortical Lesions. Dans M. Paradis (dir.), Aspects of bilingual aphasia (p. 139-176). Oxford : Pergamon Press.

GADET, Françoise. (2007). La variation sociale en français (éd. 2003). Paris : Ophrys.

Grosjean, François. (1993). Le bilinguisme et le biculturalisme. Essai et définition. Tranel, 19, 13-41.

Gumperz, John. (1971). Linguistic and Social Interaction in Two Communities. Dans J. Gumperz, Language in Social Groups (chap. 9, p. 151177). Standford, CA : Standford University Press. 
Gumperz, John. (2005). Engager la conversation (éd. 1989). Paris : Éditions de minuits.

Gumperz, John \& Hymes, Dell. H. (1972). The Ethnography of Communication. États-Unis : Holt, Rinehart \& Winston Inc.

Hymes, Dell. H. (1980). Modèles pour l'interaction du langage et de la vie sociale. Études de linguistique appliquée, 37, 127-153.

Hymes, Dell. H. (2009). Vers la compétence de communication (éd. 1973, trad. 1984). Paris : Crédif-Didier.

Kim, Karl. H. S., Relkin, Norman. R., Lee, Kyoung-Min \& Hirsch, Joy. (1997). Distinct Cortical Areas Associated with Native and Second Languages. Nature, 388, 171-174.

LÜDI, Georges. (1987). Devenir Bilingue-Parler bilingue. Université de Neuchâtel : De Gruyter.

LÜDI, Georges. (2000). Synthèse : construction des répertoires pluriels dans l'interaction. Notions en Questions, 4, 179-189.

MoIRAnD, Sophie. (2003, avril). Quelles catégories descriptives pour la mise au jour des genres du discours. Communication présentée à la journée scientifique «Les genres de l'oral», Lyon, Université LumièreLyon 2. Disponible en ligne sur <http:/gric.univ-lyon2.fr/Equipe1/actes/ journees_genre.htm>.

Murphy-Lejeune, Elizabeth \& Zarate, Geneviève. (2003). L'acteur social pluriculturel : évolution politique, positions didactiques. Le français dans le monde, numéro spécial, 32-46.

Noyau, Colette \& Porquier, Rémy. (1984). Communiquer dans la langue de l'autre. Saint-Denis : Presses universitaires de Vincennes.

Perani, Daniela, Paulesu, Eraldo, Sebastian Galles, Nuria, Dupoux, Emmanuel, Dehaene, Stanislas, Bettinardi, Valentino ... Mehler, Jacques (1998). The Bilingual Brain. Proficiency and Age of Acquisition of the Second Language. Brain, 121, 1841-1852.

Portine, Henri. (2010). La notion de position énonciative : sur la question du sujet-parlant. Le français dans le monde, 48, 123-134.

Py, Bernard. (1991). Bilinguisme, exolinguisme et acquisition : rôle de L1 dans l'acquisition de L2. Tranel, 17, 147-161.

Py, Bernard. (2004). Pour une approche linguistique des représentations sociales. Langages, 154, 6-19.

Py, Bernard \& Alber Jean-Luc. (1984). Interlangue et conversation exolingue. Bulletin de la Section de linguistique de Lausanne, 6, 217-231.

Rosen, Evelyne. (2005). La mort annoncée des "quatre compétences". Pour une prise en compte du répertoire communicatif des apprenants en classe de FLE. Glottopol, 6, 120-133.

Vygotski, Lev. (1997). Pensée et langage (éd. 1934). Paris : La dispute. 